Review

\title{
Updated recommendations on the management of gastrointestinal disturbances during iron chelation therapy with Deferasirox in transfusion dependent patients with myelodysplastic syndrome - Emphasis on optimized dosing schedules and new formulations
}

\author{
Florian Nolte ${ }^{\mathrm{a}, *}$, Emanuele Angelucci ${ }^{\mathrm{b}}$, Massimo Brecciac ${ }^{\mathrm{c}}$, Norbert Gattermann ${ }^{\mathrm{d}}$, \\ Valeria Santini ${ }^{\mathrm{e}}$, Norbert Vey ${ }^{\mathrm{f}}$, Wolf-Karsten Hofmann ${ }^{\mathrm{a}}$ \\ a Department of Hematology and Oncology, University Hospital Mannheim, Medical Faculty Mannheim of the University of Heidelberg, Germany \\ ${ }^{\mathrm{b}}$ Hematology and Bone Marrow Transplant Unit, and Medical Oncology Department, Ospedale Oncologico “Armando Businco", Cagliari, Italy \\ c Department of Cellular Biotechnologies and Hematology, "La Sapienza" University, Rome, Italy \\ ${ }^{\mathrm{d}}$ Comprehensive Cancer Center and Department of Hematology, Oncology, and Clinical Immunology, Heinrich Heine University, Düsseldorf, Germany \\ e Division of Hematology, University of Florence, Florence, Italy \\ f Department of Hematology, Institute Paoli Calmettes, Marseille, France
}

\section{A R T I C L E I N F O}

\section{Article history:}

Received 19 March 2015

Received in revised form 4 June 2015

Accepted 16 June 2015

Available online 20 June 2015

\section{Keywords:}

Myelodysplastic syndrome

Transfusion

Iron overload

Chelation

Gastrointestinal disturbances

Deferasirox

\begin{abstract}
A B S T R A C T
Myelodysplastic syndromes (MDS) are oligoclonal hematopoietic disorders characterized by peripheral cytopenias with anemias being the most prevalent feature. The majority of patients will depend on regular transfusions of packed red blood cells (PRBC) during the course of the disease. Particularly patients with MDS and low risk for transformation into acute myeloid leukemia and low risk of early death will receive PRBC transfusions on a regular basis, which puts them at high risk for transfusional iron overload. Transfusion dependence has been associated with negative impact on organ function and reduced life expectancy.

Recently, several retrospective but also some prospective studies have indicated, that transfusion dependent patients with MDS might benefit from consequent iron chelation with regard to morbidity and mortality. However, low treatment adherence due to adverse events mainly gastrointestinal in nature is an important obstacle in achieving sufficient iron chelation in MDS patients. Here, we will summarize and discuss the existing data on Deferasirox in low risk MDS published so far and provide recommendations for optimal management of gastrointestinal adverse events during iron chelation aiming at improving treatment compliance and, hence, sufficiently removing excess iron from the patients.
\end{abstract}

(C) 2015 Published by Elsevier Ltd.

\section{Contents}

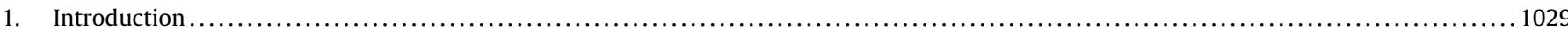

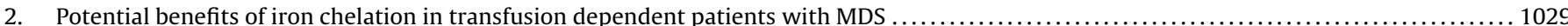

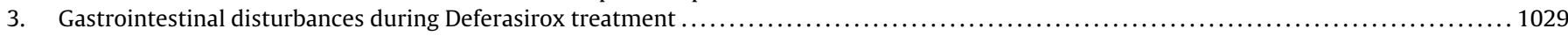



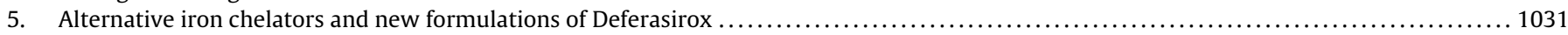

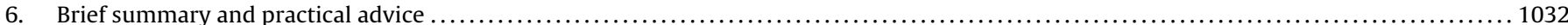

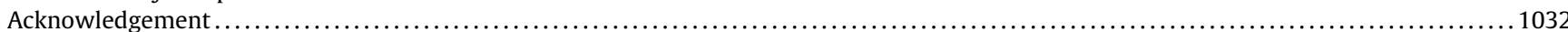

References....

* Corresponding author at: Department of Hematology and Oncology, University Hospital Mannheim, Medical Faculty of the University of Heidelberg, Theodor-Kutzer-Ufer 1-3, D-68167 Mannheim, Germany.

E-mail address: florian.nolte@medma.uni-heidelberg.de (F. Nolte). 


\section{Introduction}

Myelodysplastic syndromes (MDS) are bone marrow failures that show an increasing incidence in the elderly [1]. They are recognized as oligoclonal hematopoietic disorders characterized by peripheral cytopenias with anemias being the most prevalent cytopenic feature. The majority of patients is either transfusion dependent for red blood cells or becomes transfusion dependent during the course of the disease. Particularly patients with MDS and low risk for transformation into acute myeloid leukemia and low risk of early death will receive packed red blood cell (PRBC) transfusions on a regular basis, which puts them at high risk for transfusional iron overload (IOL) since the human organism has no natural means of removing excess iron. Transfusion dependence has been associated with negative impact on organ function and reduced life expectancy [2,3], though data are conflicting [4].

IOL leads to deposition of iron in organs such as the liver, the heart and the endocrine glands. In patients with inherited anemias such as patients suffering from thalassemia or sickle cell disease, repetitive transfusions and consecutive IOL can cause organ damage leading to liver cirrhosis, cardiac failure or diabetes mellitus [5-7].

In addition to transfusion associated IOL, some patients with MDS exhibit high ferritin levels although they have never received any transfusions, indicating a disturbed iron metabolism. Advances in the understanding of iron physiology and pathophysiology suggest, that insufficient erythropoiesis in MDS patients leads to decreased hepcidin levels and consecutively increased intestinal iron absorption. In fact, the recently discovered erythroid regulator erythroferrone links aberrant erythropoiesis to hepcidin suppression with consecutive increased dietary iron absorption [8]. Of note, erythroid progenitors in MDS patients show a particularly high expression of erythroferrone, which provides a plausible explanation, that IOL can be evident in MDS patients, who never received any transfusions of PRBC [9].

The introduction of efficacious iron chelation with deferioxamine (DFO) improved the outcome of patients with transfusion dependent anemias such as thalassemia and sickle cell disease dramatically $[5,6]$. This might be mainly due to the elimination of the so-called labile plasma iron (LPI), which catalyzes the generation of reactive oxygen species (ROS). ROS exert a high reactivity with cellular organelles and structures such as the plasma membrane, mitochondria, intracellular enzymes and nucleic acids inducing cellular dysfunction and cell death [10].

Although DFO has demonstrated high efficacy in treatment of IOL continuous and subcutaneous administration is necessary to provide sufficient coverage of LPI due to the short plasma half-life of DFO. It has been shown that this way of application is a major obstacle for providing a sufficient iron chelation (IC). Patients are affected by the inconvenient administration, which subsequently leads to lower adherence to the treatment and insufficient IC resulting in increased morbidity and mortality [5,6]. Interestingly, IC has been shown to improve hematopoiesis in some patients [11-13]. Although the exact mechanism is not clear yet improvement of hematopoiesis might be due to a reduction of oxidative stress in hematopoietic progenitors in general and erythropoietic progenitors in particular [10].

Deferasirox (DFX) is an orally administered iron chelator, which has demonstrated high efficacy in reduction of iron burden in patients with transfusional IOL. The plasma half-life of DFX is approximately $13 \mathrm{~h}$, which allows a once daily administration providing $24 \mathrm{~h}$ coverage of LPI [14]. It showed a similar efficacy in lowering iron burden as compared to DFO in MDS patients $[15,16]$.

Recently, several retrospective but also some prospective studies have indicated, that transfusion dependent patients with MDS might benefit from consequent iron chelation with regard to morbidity and mortality [17-24]. However, gastrointestinal adverse events leading to reduced quality of life in these patients are associated with low treatment adherence and are considered as a major obstacle in achieving consequent and effective iron chelation with DFX. Here, we will update our recommendations on the management of gastrointestinal disturbances during treatment of iron-overloaded patients with MDS using DFX [25].

\section{Potential benefits of iron chelation in transfusion dependent patients with MDS}

It is well established that IOL has a deleterious effect in patients with inherited anemia, i.e. thalassemia and sickle cell disease. Introduction of iron chelation significantly improved survival in those patients by preventing particularly cardiac failure, but also hepatic and pancreatic damage and pituitary insufficiency [5,6]. The effect of IOL in MDS patients, however, is controversial. The pros and cons for and against iron chelation range from consideration of excess iron as a toxin that needs to be removed on the one hand and regarding the iron issue as a paranoia on the other hand [26-28]. Advocates for initiation of iron chelation refer to the usually older age of MDS patients presenting with clinical significant comorbidities, which render them more prone to iron-related organ toxicity. In contrast, the opponents consider the effect of IOL clinically less relevant due to the reduced life expectancy in anemic patients with MDS as compared to patients with other anemias.

There is evidence, that transfusion dependency and development of IOL might have a negative impact on patient survival $[2,29]$. Consequently, several retrospective and observational studies have suggested a beneficial influence of iron chelation on mortality and morbidity in MDS patients with transfusional IO [17-24].

DFX has shown its efficacy in sufficiently reducing serum ferritin in transfusion dependent patients with MDS and IOL [30-34]. Although mainly obtained from retrospective trials, data indicate that DFX might improve survival and reduce morbidity in such patients. Moreover, improvement of hematopoiesis during DFX treatment was reported by several groups with erythroid, neutrophil and platelet responses seen in 6-21\%, 3-17\%, and 15-30\% of the patients, respectively, including transfusion independency for PRBC at 12 months of DFX treatment of $12 \%$ in a trial from the Italian GIMEMA group [33-35].

However, since it is likely that physicians estimate a patient's prognosis not only on the basis of disease-related risk scores but also according to the patient's general fitness, there might be a systematic bias in all of the aforementioned studies, because it cannot be excluded that in patients with a better performance status iron chelation may have been more likely to be initiated. This circumstance has recently been emphasized in a Cochrane analysis, which also underlined the need for well controlled randomized trials [36].

\section{Gastrointestinal disturbances during Deferasirox treatment}

Although DFX is highly effective in removing excess iron from iron overloaded MDS patients, high dropout rates of approximately $50 \%$ of patients within one year were observed in the majority of clinical studies [30,32-34]. The main reasons for discontinuation were adverse events, gastrointestinal in nature in the majority of patients as discussed below.

Gattermann et al. reported, that of 327 MDS patients 66\% experienced adverse events, that were considered to be drug related. Gastrointestinal events were the most frequent AEs with diarrhea, nausea, vomiting and abdominal pain occurring in $33 \%$, 
$13 \%, 8 \%$, and $8 \%$, of the patients, respectively. Of 341 enrolled patients, 25 patients discontinued the DFX treatment due to gastrointestinal AEs [30]. In the one year GIMEMA trial, $43 \%$ of the patients completed the trial. The main reason for discontinuation were adverse events in $33 \%$ of the patients and of the treatment related adverse events $45 \%$ were gastrointestinal in nature [34]. Although adverse events were mild to moderate in the majority of cases, they limited the adherence of the patients to a consequent and sufficient treatment schedule.

Interestingly, subgroup analyses of the EPIC cohort revealed that GI disturbances were more frequent in the MDS patient cohort as compared to patients with inherited anemias such as thalassemia, which might be an indication of a higher susceptibility of the elderly patients to DFX with regard to GI disturbances.

Taken together, to provide a sufficient coverage of iron overloaded MDS patients with DFX, effective prevention and treatment of gastrointestinal adverse events is crucial.

\section{Management of gastrointestinal adverse events}

On July 18th 2014 an expert panel had an extensive discussion regarding optimal dosing schedules and procedures to manage gastrointestinal disturbances that frequently impair the success of iron chelation in iron overloaded MDS patients using DFX. The panel agreed that gastrointestinal disturbances interfere with the daily routines of the patients and affect their quality of life and should therefore be prevented as far as possible. If adverse events cannot be prevented they should be treated effectively. Prior to treatment initiation patients should be informed in detail about the necessity of the treatment and the possible adverse events, particularly gastrointestinal disturbances.

Although no data from clinical trials do exist, that initiating the treatment at low DFX doses and increasing the dose according to tolerability and efficacy might prevent gastrointestinal disturbances, the panel agreed that this might be a helpful strategy to start chelation with DFX. The drug should be initiated at a flat dose of $500 \mathrm{mg}$ once daily. If tolerability is good, the dose should be increased weekly by $500 \mathrm{mg}$ per day to the target dose, which should be calculated on the basis of the serum ferritin level and transfusion frequency. For patients with a serum ferritin $>1000 \mathrm{ng} / \mathrm{L}$, who receive more than 2 units PRBC per 4 weeks but less than 2 units per 2 weeks, the target dose should be $30 \mathrm{mg} / \mathrm{kg}$ body weight per day. For patients with a higher transfusion frequency and high serum ferritin levels who do not respond to a dose of $30 \mathrm{mg} / \mathrm{kg}$ body weight, even target doses of $40 \mathrm{mg} / \mathrm{kg}$ body weight might be necessary to reduce body iron. For iron overloaded patients with transfusion frequencies of less than 2 units PRBC, DFX
Table 1

Recommendations for prevention of gastrointestinal disturbances.

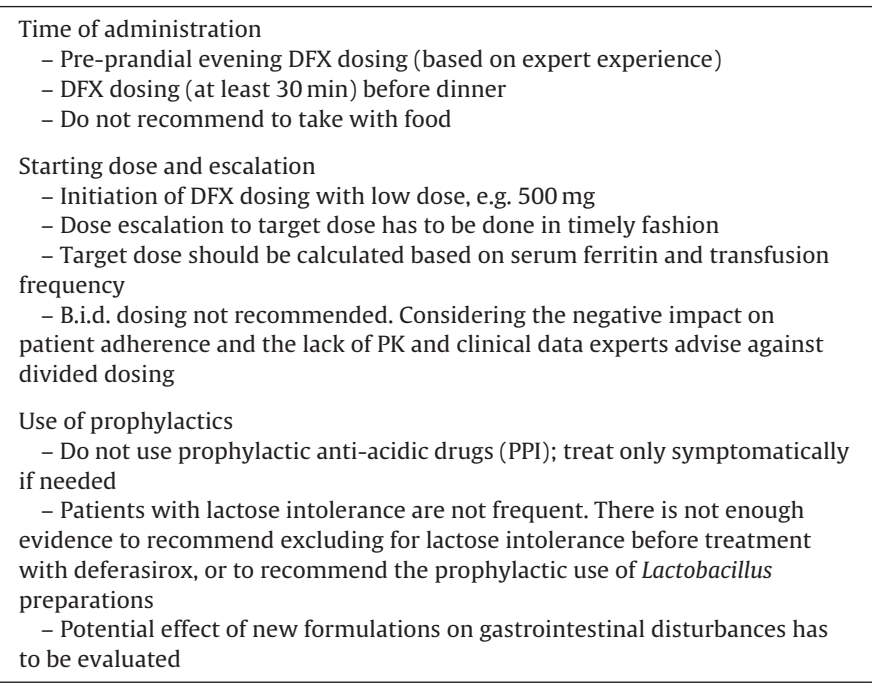

doses of $20 \mathrm{mg} / \mathrm{kg}$ body weight might be enough to reduce body iron (see Fig. 1). Of note, patients should be closely followed-up with regular determination of body iron status, to avoid undertreatment (but also over-treatment) by failing to achieve sufficient and adequate drug doses to eliminate iron from the body either as "induction" therapy or maintenance. For a summary of recommendations for the prevention of gastrointestinal disturbances see Table 1 . In addition to these recommendations, it might be beneficial in some patients to try different solvents such as water or juices to find out the optimal composition for intake of DFX. Moreover, to achieve a thorough dissolution of DFX in the chosen solvent, electronic dispersers might be helpful.

Pre-prandial evening dosing still might be a reasonable approach. Even if it does not reduce the incidence of gastrointestinal disturbances in all cases, it might prevent interference with daily routines of the patients by virtue of the GI disturbances being at night.

For the management of diarrhea during DFX treatment the panel recommends the algorithm shown in Fig. 2, which is a slightly modified version of the initial algorithm, i.e. administration of low doses pre-prandial in the evening should be the starting schedule and is not considered the alternative procedure, if the target morning doses are not tolerated. For practical reasons a simple guidance using the frequency of bowel movements has been applied in the diarrhea management algorithm.

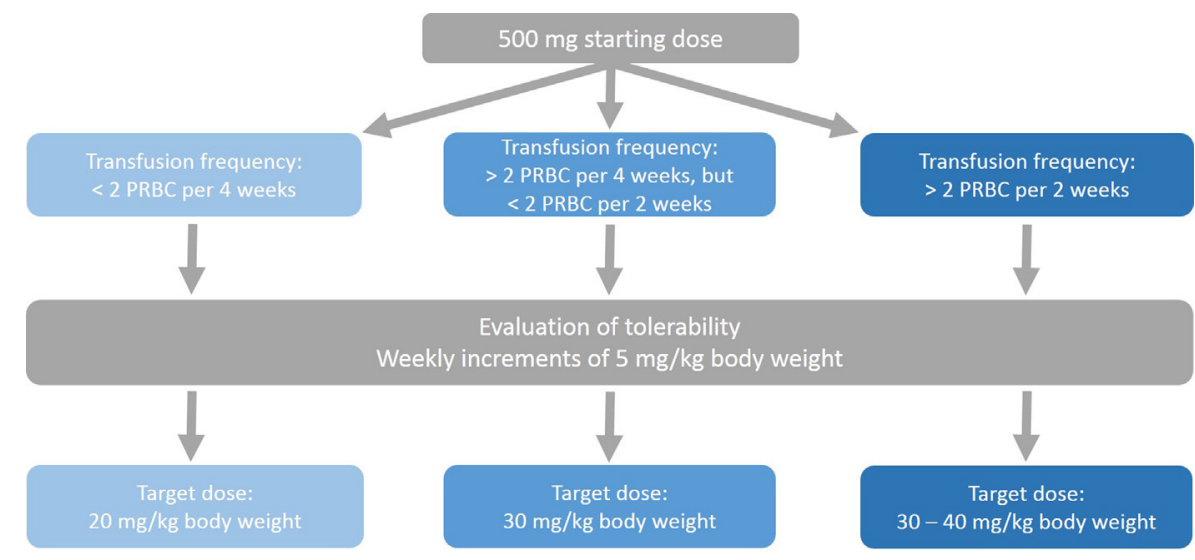

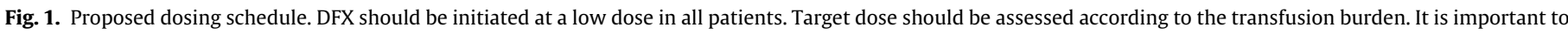
achieve the target dose in a timely manner. 


\begin{tabular}{|c|c|c|}
\hline Mild (episodes) & Moderate (episodes) & Severe (episodes) \\
\hline$<4$ & $4-6$ & $>6$ \\
\hline $\begin{array}{l}\text { Diarrhea „MILD“ - } \\
\text { Supportive Care }\end{array}$ & $\begin{array}{c}\text { Diarrhea „MODERATE“ - } \\
\text { Supportive Care and } \\
\text { Dose Modification }\end{array}$ & $\begin{array}{c}\text { Diarrhea „SEVERE“ - } \\
\text { Supportive Care and } \\
\text { Dose Suspension }\end{array}$ \\
\hline 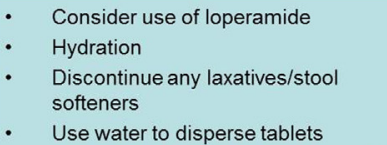 & $\begin{array}{l}\text { - Loperamide at standard doses } \\
\text { - Other supportive care as descibred } \\
\text { in "MILD“ recommendations } \\
\text { - } \quad \text { Reduce DFX dose to } 10 \mathrm{mg} / \mathrm{kg} / \text { day }\end{array}$ & $\begin{array}{l}\text { - Supportive care as described in } \\
\text { "MILD" recommendations } \\
\text { Hold DFX dose }\end{array}$ \\
\hline $\begin{array}{l}\text { Diarrhea resolved } \\
\text { - Continue hydration } \\
\text { Discontinue loperamide after } \\
\text { 12h diarrhea-free period }\end{array}$ & $\begin{array}{l}\text { Diarrhea resolved } \\
\text { - } \quad \text { Continue hydration } \\
\text { - } \quad \text { Discontinue loperamide after } \\
\text { 12h diarrhea-free period } \\
\text { - } \quad \text { Re-escalate DFX dose to }\end{array}$ & $\begin{array}{l}\text { Diarrhea resolved } \\
\text { - } \quad \text { Continue hydration } \\
\text { Discontinue loperamide after } \\
12 \mathrm{~h} \text { diarrhea-free period } \\
\text { Re-initiate DFX dose at } 10 \\
\mathrm{mg} / \mathrm{kg} / \text { day and dose adjust in } \\
\text { increments of } 5 \mathrm{mg} / \mathrm{kg} \text { each } \\
\text { week to target dose }\end{array}$ \\
\hline $\begin{array}{l}\text { Diarrhea stable or worse after } 1 \text { week } \\
\text { - Follow "MODERATE" algorithm }\end{array}$ & $\begin{array}{l}\text { Diarrhea stable or worse after } 1 \text { week } \\
\text { - Follow "SEVERE" algorithm }\end{array}$ & $\begin{array}{l}\text { Diarrhea stable or worse after } 1 \text { week } \\
\text { - Investigate other etiologies for } \\
\text { diarrhea } \\
\text { Discontinue DFX therapy if } \\
\text { diarrhea is unmanageable on re- } \\
\text { challenge }\end{array}$ \\
\hline
\end{tabular}

Fig. 2. Algorithm for management of diarrhea.

The symptom "abdominal pain" requires an exact diagnosis since management of upper and lower abdominal pain differs. Switching a morning dose to an evening pre-prandial dosing should be considered. Dosing before bedtime is not recommended due to the risk of oesophageal irritation and bleeding. In cases of persisting mild-to-moderate abdominal pain dose reductions should be considered before treatment interruptions. Reduction should be done in steps of $5 \mathrm{mg} / \mathrm{kg} /$ day. If abdominal pain has resolved DFX dose should be increased in $5 \mathrm{mg} / \mathrm{kg} /$ day steps to the target dose. Use of antacids for upper abdominal pain might be considered. Spasmolytic drugs might be helpful for lower abdominal pain. However, analgesic drugs and non-steroidal anti-inflammatory drugs are not recommended due to the common GI side effects of these drugs. Temporary interruption of DFX treatment can be considered in case of persisting severe abdominal pain until symptoms resolve. DFX should then be reinitiated and the dose should be escalated in $5 \mathrm{mg} / \mathrm{kg} /$ day steps. Of course, serious reasons for severe abdominal pain should be ruled out. Recommendations are summarized in Table 2.

Antiemetics such as metoclopramide can be used in case of nausea and vomiting. Since vomiting is the only AE which does not seem to improve with long-term use, pre-prandial administration of DFX in the evening should be considered especially if vomiting (and nausea) are related to the morning dose. In addition, administration of the drug in the evening prevents, that gastrointestinal disturbances interfere with the daily routines of the patients. Reduction of DFX in steps of $5 \mathrm{mg} / \mathrm{kg} /$ day should be done in cases of severe nausea/vomiting. Re-initiation of DFX and increase on target should be done when symptoms have resolved. Recommendations are summarized in Table 3.

\section{Alternative iron chelators and new formulations of Deferasirox}

In case DFX is judged inadequate in a patient, other chelators can be considered. As mentioned above, DFO has shown efficacy in patients with MDS and IOL. Nevertheless, treatment adherence to
Table 2

Recommendations for management of abdominal pain Patients with constipation have to be managed by another algorithm!

Diagnosis is important

- Management of upper abdominal pain differs from lower abdominal pain (with constipation and/or diarrhea and/or bloating)

Time of administration

- Anecdotal reports of benefit: patient is advised to refrain from solid foods for $2 \mathrm{~h}$ after deferasirox

- Do not recommend DFX dosing before bedtime

Use of medication

- For upper abdominal pain, use anti-acidic drugs

- Consider using spasmolytic drugs

- Do not use narcotic pain medications and non-steroidal anti-inflammatory drugs

Dosing

- Mild-to-moderate cases, consider DFX dose reduction before treatment interruptions

- Reduce DFX dose in steps of $5 \mathrm{mg} / \mathrm{kg} /$ day

- Increase DFX dosing in steps of $5 \mathrm{mg} / \mathrm{kg} /$ day on target when abdominal pain has been resolved

- Severe cases: temporarily interrupt deferasirox until abdominal pain has been resolved. Restart at low dose and increase in steps of $5 \mathrm{mg} / \mathrm{kg} / \mathrm{day}$ on target.

Table 3

Recommendations for management of nausea/vomiting.

\footnotetext{
Vomiting considered as a severe expression of nausea

- Management of both similar

Use anti-emetics (metoclopramide)

Time of administration

- Strong notion that nausea (especially vomiting) might be related to the morning dosing, especially since vomiting is the only AE that does not show a trend toward reduction with long-term use

Reduce DFX dosing in steps of $5 \mathrm{mg} / \mathrm{kg} /$ day in case of severe nausea/vomiting

- Increase dosing in steps of $5 \mathrm{mg} / \mathrm{kg} /$ day on target when nausea/vomiting has been resolved
} 
DFO often is hampered by the inconvenience of its administration i.e. continuous subcutaneous application for $24 \mathrm{~h}$ a day for several days. Deferiprone is another oral available iron chelator that is approved for treatment of IOL. Although it has shown its efficacy in removing excess iron, Deferiprone is rather considered as a third line drug, due to its side effects particularly induction of neutropenia and agranulocytosis, respectively. However, Deferiprone can be considered if DFX or DFO cannot be applied to a patient, but close monitoring of differential blood counts should be done. A review on the use of the different iron chelators in the treatment of IOL in MDS patients has been published recently [37].

Although the exact mechanisms of gastrointestinal intolerance are not yet fully understood, osmotic mechanisms might play a role in the development of diarrhea. Moreover, since DFX contains lactose, patients with lactose intolerance might be more susceptible to GI disturbances such as diarrhea.

A new formulation of DFX as a film coated tablet (FCT) has been developed and is currently under investigation in clinical trials. The FCT contains the same active substance as the dispersible formulation but lacks lactose and sodium sulphate that have been implicated in the induction of gastrointestinal disturbances. Moreover, pharmacokinetic studies demonstrated a superior bioavailability of the FCT as compared to the dispersible form with $360 \mathrm{mg}$ of the FCT equating to $500 \mathrm{mg}$ of dispersible DFX. Interestingly, in contrast to DFX in the dispersible form, FCT seems to lack a food effect with regard to its bioavailability, which might facilitate administration of the drug and lead to a more predictable drug exposure.

\section{Brief summary and practical advice}

- Data regarding IC in MDS patients are controversial, but multiple analyses suggest a benefit including erythroid improvement and transfusion independence.

- Patients should be informed in detail about the nature of gastrointestinal disturbances.

- DFX dosing should be done pre-prandial in the evening for patients with GI side effects.

- DFX should be initiated at low dose levels, e.g. $500 \mathrm{mg}$ per day.

- Escalation of DFX dose to target dose should be done in a timely fashion.

- Target dose should be calculated based on serum ferritin and transfusion frequency.

- New formulations might improve tolerability and adherence to treatment.

\section{Acknowledgement}

We thank Christiane Schumann for critically reviewing the manuscript.

\section{References}

[1] U. Germing, C. Strupp, A. Kundgen, et al., No increase in age-specific incidence of myelodysplastic syndromes, Haematologica 89 (8) (2004) 905-910.

[2] L. Malcovati, M.G. Porta, C. Pascutto, et al., Prognostic factors and life expectancy in myelodysplastic syndromes classified according to WHO criteria: a basis for clinical decision making, J. Clin. Oncol. 23 (30) (2005) 7594-7603

[3] M. Takatoku, T. Uchiyama, S. Okamoto, et al., Retrospective nationwide survey of Japanese patients with transfusion-dependent MDS and aplastic anemia highlights the negative impact of iron overload on morbidity/mortality, Eur. J. Haematol. 78 (6) (2007) 487-494.

[4] C.E. Chee, D.P. Steensma, W. Wu, C.A. Hanson, A. Tefferi, Neither serum ferritin nor the number of red blood cell transfusions affect overall survival in refractory anemia with ringed sideroblasts, Am. J. Hematol. 83 (8) (2008) 611-613.
[5] B. Modell, M. Khan, M. Darlison, Survival in beta-thalassaemia major in the UK: data from the UK Thalassaemia Register, Lancet 355 (9220) (2000) 2051-2052.

[6] C. Borgna-Pignatti, S. Rugolotto, P. De Stefano, et al., Survival and complications in patients with thalassemia major treated with transfusion and deferoxamine, Haematologica 89 (10) (2004) 1187-1193.

[7] P. Brissot, M. Ropert, C. Le Lan, O. Loreal, Non-transferrin bound iron: a key role in iron overload and iron toxicity, Biochim. Biophys. Acta 1820 (3) (2012) $403-410$.

[8] L. Kautz, G. Jung, E.V. Valore, S. Rivella, E. Nemeth, T. Ganz, Identification of erythroferrone as an erythroid regulator of iron metabolism, Nat. Genet. 46 (7) (2014) 678-684.

[9] M. Mossner, A. Stöhr, F. Nolte, et al., Gene expression of the erythroid regulator erythroferrone (ERFE) is highly deregulated in CD71+ erythroprogenitor cells of patients with myelodysplastic syndromes and demonstrates prognostic relevance, Blood 124 (21) (2014) 4620.

[10] N. Gattermann, E.A. Rachmilewitz, Iron overload in MDS-pathophysiology, diagnosis, and complications, Ann. Hematol. 90 (1) (2011) 1-10.

[11] P.D. Jensen, I.M. Jensen, J. Ellegaard, Desferrioxamine treatment reduces blooc transfusion requirements in patients with myelodysplastic syndrome, Br. J. Haematol. 80 (1) (1992) 121-124.

[12] P.D. Jensen, L. Heickendorff, B. Pedersen, et al., The effect of iron chelation on haemopoiesis in MDS patients with transfusional iron overload, Br. J. Haematol. 94 (2) (1996) 288-299.

[13] R. Guariglia, M.C. Martorelli, O. Villani, et al., Positive effects on hematopoiesis in patients with myelodysplastic syndrome receiving deferasirox as oral iron chelation therapy: a brief review, Leuk. Res. 35 (5) (2011) 566-570.

[14] P.L. Greenberg, C.A. Koller, Z.I. Cabantchik, et al., Prospective assessment of effects on iron-overload parameters of deferasirox therapy in patients with myelodysplastic syndromes, Leuk. Res. 34 (12) (2010) 1560-1565.

[15] M.D. Cappellini, A. Cohen, A. Piga, et al., A phase 3 study of deferasirox (ICL670), a once-daily oral iron chelator, in patients with beta-thalassemia, Blood 107 (9) (2006) 3455-3462.

[16] A. Piga, R. Galanello, G.L. Forni, et al., Randomized phase II trial of deferasirox (Exjade, ICL670), a once-daily, orally-administered iron chelator, in comparison to deferoxamine in thalassemia patients with transfusional iron overload, Haematologica 91 (7) (2006) 873-880.

[17] C. Rose, S. Brechignac, D. Vassilief, et al., Does iron chelation therapy improve survival in regularly transfused lower risk MDS patients? A multicenter study by the GFM (Groupe Francophone des Myelodysplasies), Leuk. Res. 34 (7) (2010) 864-870.

[18] A. Raptis, M.S. Duh, S.T. Wang, et al., Treatment of transfusional iron overload in patients with myelodysplastic syndrome or severe anemia: data from multicenter clinical practices, Transfusion (Paris) 50 (1) (2010) 190-199.

[19] R.S. Komrokji, N.H.A. Ali, E. Padron, J.E. Lancet, A.F. List, Impact of iron chelation therapy on overall survival and AML transformation in lower risk MDS patients treated at the Moffitt Cancer Center, Blood 118 (21) (2011) 2776.

[20] R.M. Lyons, B.J. Marek, C. Paley, et al., Relationship between chelation and clinical outcomes in 600 lower-risk mds patients: registry analysis at 36 months, Blood 120 (21) (2012) 3800

[21] J. Neukirchen, F. Fox, A. Kundgen, et al., Improved survival in MDS patients receiving iron chelation therapy - a matched pair analysis of 188 patients from the Dusseldorf MDS registry, Leuk. Res. 36 (8) (2012) 1067-1070.

[22] A.F. Remacha, B. Arrizabalaga, A. Villegas, et al., Evolution of iron overload in patients with low-risk myelodysplastic syndrome: iron chelation therapy and organ complications, Ann. Hematol. 94 (5) (2015) 779-787.

[23] M. Delforge, D. Selleslag, Y. Beguin, et al., Adequate iron chelation therapy for at least six months improves survival in transfusion-dependent patients with lower risk myelodysplastic syndromes, Leuk. Res. 38 (5) (2014) 557-563.

[24] H.A. Leitch, C.S. Leger, T.A. Goodman, et al., Improved survival in patients with myelodysplastic syndrome receiving iron chelation therapy, Clin. Leuk. 2 (3) (2008) 205-211.

[25] F. Nolte, E. Angelucci, P. Beris, et al., Clinical management of gastrointestinal disturbances in patients with myelodysplastic syndromes receiving iron chelation treatment with deferasirox, Leuk. Res. 35 (9) (2011)1131-1135.

[26] A.F. List, Iron overload in myelodysplastic syndromes: diagnosis and management, Cancer Control 17 (2010) 2-8.

[27] D.P. Steensma, Myelodysplasia paranoia: iron as the new radon, Leuk. Res. 33 (9) (2009) 1158-1163.

[28] I.H. Kim, J.H. Moon, S.N. Lim, et al., Efficacy and safety of deferasirox estimated by serum ferritin and labile plasma iron levels in patients with aplastic anemia, myelodysplastic syndrome, or acute myeloid leukemia with transfusional iron overload, Transfusion 55 (July (7)) (2015) 1613-1620.

[29] G. Sanz, B. Nomdedeu, E. Such, et al., Independent impact of iron overload and transfusion dependency on survival and leukemic evolution in patients with myelodysplastic syndrome, ASH Annu. Meet. Abstr. 112 (11) (2008) 640.

[30] N. Gattermann, C. Finelli, M.D. Porta, et al., Deferasirox in iron-overloaded patients with transfusion-dependent myelodysplastic syndromes: results from the large 1-year EPIC study, Leuk. Res. 34 (9) (2010) 1143-1150.

[31] N. Gattermann, A. Jarisch, R. Schlag, et al., Deferasirox treatment of iron-overloaded chelation-naive and prechelated patients with myelodysplastic syndromes in medical practice: results from the observational studies eXtend and eXjange, Eur. J. Haematol. 88 (3) (2012) 260-268. 
[32] A.F. List, M.R. Baer, D.P. Steensma, et al., Deferasirox reduces serum ferritin and labile plasma iron in RBC transfusion-dependent patients with myelodysplastic syndrome, J. Clin. Oncol. 30 (17) (2012)

2134-2139.

[33] F. Nolte, B. Hochsmann, A. Giagounidis, et al., Results from a 1-year, open-label, single arm, multi-center trial evaluating the efficacy and safety of oral Deferasirox in patients diagnosed with low and int-1 risk

myelodysplastic syndrome (MDS) and transfusion-dependent iron overload, Ann. Hematol. 92 (2) (2013) 191-198.

[34] E. Angelucci, V. Santini, A.A. Di Tucci, et al., Deferasirox for

transfusion-dependent patients with myelodysplastic syndromes: safety, efficacy, and beyond (GIMEMA MDS0306 Trial), Eur. J. Haematol. 92 (6) (2014) 527-536.

[35] N. Gattermann, C. Finelli, M. Della Porta, et al., Hematologic responses to deferasirox therapy in transfusion-dependent patients with myelodysplastic syndromes, Haematologica 97 (9) (2012) 1364-1371.

[36] J.J. Meerpohl, L.K. Schell, G. Rucker, et al., Deferasirox for managing iron overload in people with myelodysplastic syndrome, Cochrane Database Syst. Rev. 10 (2014), CD007461.

[37] S. Temraz, V. Santini, K. Musallam, A. Taher, Iron overload and chelation therapy in myelodysplastic syndromes, Crit. Rev. Oncol. Hematol. 91 (1) (2014) 64-73. 